\title{
A Probabilistic Approach to a Differential-Difference Equation Arising in Analytic Number Theory
}

\author{
By Jean-Marie-François Chamayou
}

Abstract. The differential-difference equation

$$
\begin{aligned}
t v^{\prime}(t)+v(t-1) & =0, & & t>1, \\
v(t) & =0, & & t<0, \\
v(t) & =\text { constant }, & & 0 \leqq t \leqq 1,
\end{aligned}
$$

can be solved by the Monte-Carlo method, for the initial condition $v(t)=e^{-\gamma}, 0 \leqq t \leqq 1$, where the $v(t)$ represent the probability density of a random variable:

$$
t=\lim _{n \rightarrow \infty} \sum_{i=1}^{n} \prod_{j=1}^{i} x_{j}
$$

where the $x_{i}$ are independent and uniformly distributed on $(0,1)$.

I. Introduction. The function $\psi(x, y)$ is equal to the number of integers less than or equal to $x$ and free of prime factors greater than $y$. Chowla and Vijayaraghavan, Ramaswami, Buchstab and de Bruijn have shown that [1]:

$$
\lim _{y \rightarrow \infty} \frac{\psi\left(y^{t}, y\right)}{y^{t}}=v(t)
$$

where $v(t)$ is a function satisfying

$$
\begin{aligned}
t v^{\prime}(t)+v(t-1) & =0, & & t>1, \\
v(t) & =0, & & t<0, \\
v(t) & =1, & & 0 \leqq t \leqq 1 .
\end{aligned}
$$

Many authors have studied the limits and asymptotic behaviour of this equation [2]; Norton gives an exhaustive bibliography [3]. Highly accurate numerical results were obtained by Dickman, Bellman, Van de Lune ([4], [5], [6]).

The differential-difference equation solution by the Monte-Carlo method does not claim to be as accurate as these previous calculations but only shows a probabilistic aspect of this equation.

II. Stochastic Model. Let $u_{n}$ be the random variable: $u_{n}=x_{1}+x_{1} x_{2}+\cdots+$ $x_{1} x_{2} \cdots x_{n}$, where $x_{i}$ are independent random variables uniformly distributed on $(0,1)$.

Received January 4, 1972.

AMS (MOS) subject classifications (1970). Primary 65C05, 10-04, 60K99; Secondary 34J10, $10 \mathrm{~A} 25$.

Key words and phrases. Differential-difference equation, Monte-Carlo method, stochastic processes, elementary prime number theory, explicit machine computations.

Copyright @ 1973, American Mathematical Society 
It may be deduced from the distribution of a product of $x_{i}$ variables that if $n \rightarrow \infty$, $u_{n}$ converges in probability to a limit.

Lemma. Assume that $v(t)$ is a function continuous on $0<t<\infty$ satisfying the following equation:

$$
\begin{aligned}
t v^{\prime}(t)+v(t-1) & =0, & & t>1, \\
v(t) & =0, & & t<0, \\
v(t) & =C, & & 0 \leqq t \leqq 1 .
\end{aligned}
$$

This function is identical to $f(t)$ : the probability density of a random variable:

$$
t=\lim _{n \rightarrow \infty} \sum_{i=1}^{n} \prod_{i=1}^{i} x_{i}
$$

where $x_{i}$ are independent random variables uniformly distributed on $(0,1)$ if the constant $C$ equals $e^{-\gamma}, \gamma$ being the Euler constant.

Proof.* Introduce

$$
t_{a}=\sum_{i=1}^{\infty} \prod_{j=1}^{i} x_{i} \quad \text { and } \quad t_{b}=\sum_{i=2}^{\infty} \prod_{i=2}^{i} x_{i}
$$

$t_{a}$ and $t_{b}$ have the same probability distribution and $t_{a}=x_{1}\left(1+t_{b}\right), t_{b}$ and $x_{1}$ are independent.

Let $F(t)$ be the distribution function of $t_{a}$ :

$$
F(t)=\operatorname{Pr}\left[t_{a} \leqq t\right]
$$

of course, if $t<0$, then $F(t)=0$.

If $t>0$, we have

$$
\begin{aligned}
F(t) & =\operatorname{Pr}\left[t_{a} \leqq t\right]=\operatorname{Pr}\left[x_{1}\left(t_{b}+1\right) \leqq t\right] \\
& =\sum \operatorname{Pr}\left[t_{b}+1 \leqq t / x\right] \operatorname{Pr}\left[x \leqq x_{1} \leqq x+d x\right] \\
& =\sum F\left(\frac{t}{x}-1\right) \operatorname{Pr}\left[x \leqq x_{1} \leqq x+d x\right]=\int_{0}^{1} F\left(\frac{t}{x}-1\right) d x
\end{aligned}
$$

Put $(t / x)-1=s$, then

$$
F(t)=t \int_{t-1}^{\infty} \frac{F(s)}{(s+1)^{2}} d s
$$

If $0 \leqq t \leqq 1$, then

$$
F(t)=t \int_{0}^{\infty} \frac{F(s) d s}{(s+1)^{2}}=C \cdot t
$$

where $C$ is a constant. Hence, $f(t)=F^{\prime}(t)=C$ for $0 \leqq t \leqq 1$.

If $t>1$, by differentiating once, we get

$$
f(t)=(F(t)-F(t-1)) / t \geqq 0 ;
$$

by differentiating again, we find $t f^{\prime}(t)=-f(t-1), t>1$.

* I am indebted to J. J. A. M. Brands for the correction of my initial proof. 
TABLE I

\begin{tabular}{|c|c|c|}
\hline $\mathrm{n}$ & $\begin{array}{l}\operatorname{Pr}\left(u_{n} \leqslant 1\right) \\
\text { Explicit value }\end{array}$ & $\begin{array}{c}\text { Monte-Carlo value } \\
\left(10^{5} \text { - runs }\right)\end{array}$ \\
\hline 2 & 0.69315 & 0.69416 \\
\hline 3 & 0.61428 & 0.61622 \\
\hline 4 & 0.58498 & 0.58350 \\
\hline 5 & 0.57246 & 0.57356 \\
\hline 6 & 0.56674 & 0.57016 \\
\hline 7 & 0.56404 & 0.56303 \\
\hline 8 & 0.56273 & 0.56290 \\
\hline 9 & 0.56209 & 0.56381 \\
\hline 10 & 0.56177 & 0.56030 \\
\hline$\infty$ & 0.56146 & \\
\hline
\end{tabular}

Let $h(s)$ be the Laplace transform of $f(t)$ [7]:

$$
h(s)=\left(C_{0} / s\right) \exp \left\{-E_{1}(s)\right\},
$$

where

$$
E_{1}(s)=\int_{s}^{\infty} \frac{e^{-z} d z}{z}
$$


TABLE II

\begin{tabular}{|c|c|c|c|c|}
\hline \multirow{2}{*}{$t$} & \multirow{2}{*}{$\begin{array}{c}v(t) \\
\text { Explicit value }\end{array}$} & \multirow{2}{*}{$\Delta t \stackrel{t}{=} 0.1$} & \multicolumn{2}{|c|}{ Monte-Carlo value (20 000 runs) } \\
\hline & & & Rough value & $\begin{array}{l}\text { Smooth value } \\
\text { using REINSCH's } \\
\text { (10) program }\end{array}$ \\
\hline 0 & 1 & $\begin{array}{l}0 \\
0.1-0.1 \\
0.1-0.2 \\
0.2-0.3 \\
0.3-0.4 \\
0.4-0.5 \\
0.5-0.6 \\
0.6-0.7 \\
0.7-0.8 \\
0.8-0.9 \\
0.9-1.0\end{array}$ & $\begin{array}{l}0.96801 \\
0.99206 \\
1.03391 \\
0.96890 \\
1.01788 \\
1.01432 \\
1.02233 \\
0.99206 \\
1.01343 \\
0.95733\end{array}$ & \\
\hline $\begin{array}{l}1.1 \\
1.2 \\
1.2 \\
1.4 \\
1.5 \\
1.6 \\
1.7 \\
1.8 \\
1.9\end{array}$ & $\begin{array}{l}0.9046898202 \\
0.8176784432 \\
0.7376357355 \\
0.6635277634 \\
0.5945348919 \\
0.5299963708 \\
0.4693717489 \\
0.4122133351 \\
0.3581461138\end{array}$ & $\begin{array}{l}1.0-1.1 \\
1.1-1.2 \\
1.2-1.3 \\
1.3-1.4 \\
1.4-1.5 \\
1.5-1.6 \\
1.6-1.7 \\
1.7-1.8 \\
1.8-1.9 \\
1.9-2.0\end{array}$ & $\begin{array}{l}0.95911 \\
0.91547 \\
0.81484 \\
0.69016 \\
0.58419 \\
0.57974 \\
0.50849 \\
0.43992 \\
0.37492 \\
0.31169\end{array}$ & $\begin{array}{l}0.9624 \\
0.8874 \\
0.8132 \\
0.7403 \\
0.6693 \\
0.6006 \\
0.5346 \\
0.4719 \\
0.4128 \\
0.3578\end{array}$ \\
\hline $\begin{array}{l}2.0 \\
2.1 \\
2.2 \\
2.3 \\
2.4 \\
2.5 \\
2.6 \\
2.7 \\
2.8 \\
2.9 \\
3.0 \\
3.1 \\
3.2 \\
3.3 \\
3.4 \\
3.5 \\
3.6 \\
3.7 \\
3.8 \\
3.9 \\
4.0\end{array}$ & $\begin{array}{l}0.3068528194 \\
0.2604057802 \\
0.2203571379 \\
0.1857994616 \\
0.1559912639 \\
0.1303195618 \\
0.1082724430 \\
0.08941856572 \\
0.07339158076 \\
0.05987811599 \\
0.04860838829 \\
0.03932296954 \\
0.03170344451 \\
0.02546472387 \\
0.02037177906 \\
0.01622959324 \\
0.01287543418 \\
0.01017283782 \\
0.008006872188 \\
0.006280373062 \\
0.004910925648\end{array}$ & $\begin{array}{l}2.0-2.1 \\
2.1-2.2 \\
2.2-2.3 \\
2.3-2.4 \\
2.4-2.5 \\
2.5-2.6 \\
2.6-2.7 \\
2.7-2.8 \\
2.8-2.9 \\
2.9-3.0 \\
3.0-3.1 \\
3.1-3.2 \\
3.2-3.3 \\
3.3-3.4 \\
3.4-3.5 \\
3.5-3.6 \\
3.6-3.7 \\
3.7-3.8 \\
3.8-3.9 \\
3.9-4.0 \\
4.0-4.1\end{array}$ & $\begin{array}{l}0.29031 \\
0.23510 \\
0.17810 \\
0.17098 \\
0.16208 \\
0.11132 \\
0.09172 \\
0.07748 \\
0.05699 \\
0.05076 \\
0.05165 \\
0.04186 \\
0.02583 \\
0.01514 \\
0.01603 \\
0.00980 \\
0.01069 \\
0.01514 \\
0.00801 \\
0.00534 \\
0.00178\end{array}$ & $\begin{array}{l}0.3070 \\
0.2608 \\
0.2193 \\
0.1826 \\
0.1506 \\
0.1231 \\
0.0999 \\
0.0808 \\
0.0653 \\
0.0528 \\
0.0425 \\
0.0333 \\
0.0250 \\
0.0186 \\
0.0145 \\
0.0125 \\
0.0121 \\
0.0120 \\
0.0092 \\
0.0053 \\
0.0018\end{array}$ \\
\hline
\end{tabular}

Assuming that $f(t)$ is a probability $h(0)=\int_{0}^{\infty} f(t) d t=1$, the constant $C_{0}$ equals $e^{-\gamma}$, where $\gamma$ is the Euler constant.

Since $f(t)=C$ as $t=0$, we obtain the boundary condition: $\lim _{s \rightarrow \infty} \operatorname{sh}(s)=C=e^{-\gamma}$.

From $f(t)=C$ as $t=1$, inverting Laplace transform, it may be deduced again that $f(1)=e^{-\gamma}$, so that

$$
\begin{aligned}
f(t) & =0, & & t<0, \\
f(t) & =e^{-\gamma}, & & 0 \leqq t \leqq 1, \\
f^{\prime}(t) & =-f(t-1) / t, & & t>1 .
\end{aligned}
$$


TABLE II

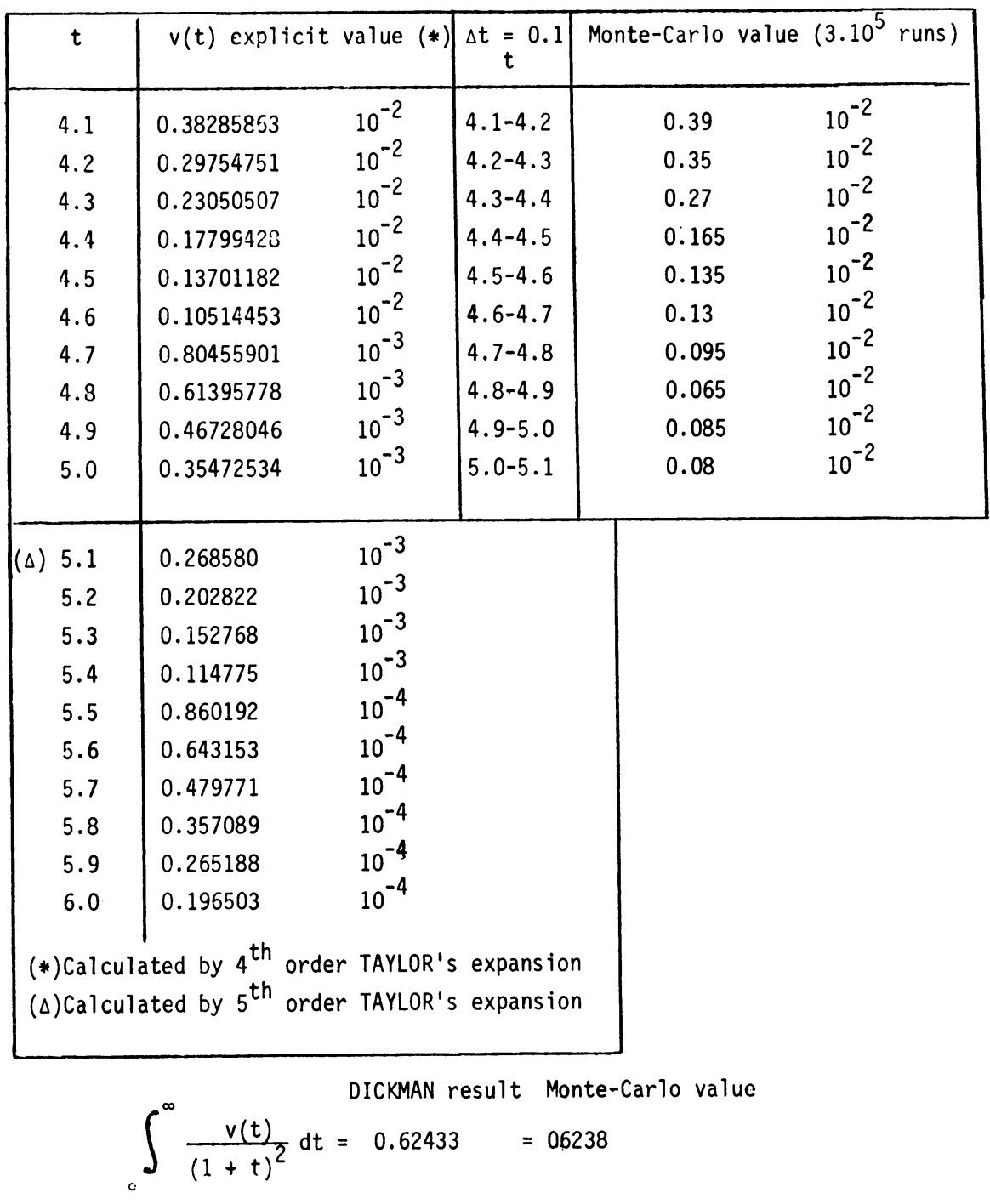

III. Numerical Calculations. For $t \leqq 4$, the solution of Eq. (1) is obtained by explicit expression (see Appendix); for $t>4$, it is impossible to express the solution by means of known functions. This explicit expression can thus be used for the wellknown equation of the statistic theory of damage [8].

$$
\begin{aligned}
t u^{\prime}(t) & =u(t-1), & & t>1, \\
u(t) & =0, & & t<0, \\
u(t) & =1, & & 0 \leqq t \leqq 1 .
\end{aligned}
$$

For $t \leqq 4$, the function $v(t)$ can be calculated with an accuracy depending solely on the polylogarithms which are used in its expression [9]. The random variable $u_{n}$ is very easy to simulate by means of the pseudo-random numbers of Lehmer's method. 
It can be seen in Section II that the $u_{n}$ distributions achieve rapid convergence as $n$ increases.

For the calculations, $n$ is chosen so that we cannot discriminate between the distributions of $u_{n}$ and $u_{n-1}$ because the statistical fluctuations of the pseudo-random numbers are greater than the discrepancy between them.

IV. Results. Table I gives an illustration of Section II; notice that we get the Euler constant simulated by $-\log \left|\operatorname{Pr}\left[u_{n} \leqq 1\right]\right|, n \rightarrow \infty$.

Table II represents the calculation of the function $v(t)$ explicitly and by simulation. Results are smoothed by the spline method [10]. Polylogarithms can be calculated by means of Chebyshev's polynomial expansion [11], [12]; Kölbig gives an excellent algorithm for the dilogarithm's calculation [13].

V. Conclusion. The main purpose of this paper is to test the ability of the Monte-Carlo method to resolve differential-difference equations, and, using a classical example, to justify further studies in the field of the statistical theory of damage and neutron transport problems [14] which involve the same mathematical data.

Acknowledgment. The computations were performed on the IBM 7044 of the Centre de Calcul Numérique de l'Université Paul Sabatier de Toulouse, using FORTRAN IV programs.

The author wishes to thank Professor D. Blanc, Directeur du Centre de Physique Atomique de l'Université Paul Sabatier, for his encouragement, and Dr. J. J. A. M. Brands, Technical Highschool, Eindhoven, Holland, for his helpful suggestions, especially concerning the correction of the proof of the lemma.

Appendix.

$v(t)$ explicit behaviour,

$$
\begin{aligned}
& v(t)=1-\log t, \quad 1 \leqq t \leqq 2 \text {, } \\
& v(t)=1-\log t+\left[\frac{1}{2} \log ^{2} t+L_{2}(1 / t)+L_{2}(-1)\right], \quad 2 \leqq t \leqq 3, \\
& v(t)=1-\log t+\left[\frac{1}{2} \log ^{2} t+L_{2}(1 / t)+L_{2}(-1)\right] \\
& -\left\{\frac{1}{4}\left[L_{3}\left(\frac{1}{4}\right)-L_{3}\left(\frac{1}{(t-1)^{2}}\right)\right]-\frac{1}{3}\left(\log ^{3}(t-1)-\log ^{3} 2\right)\right. \\
& +\frac{1}{2}\left(\log ^{2}(t-1) \log t-\log ^{2} 2 \log 3\right)+L_{2}\left(\frac{1}{t-1}\right) \log \frac{t}{t-1} \\
& -L_{2}\left(\frac{1}{2}\right) \log \left(\frac{3}{2}\right)-L_{2}\left(-\frac{1}{t-1}\right) \log (t-2)+L_{2}(-1) \log \frac{t}{3} \\
& +\left\{[\underbrace{\left.\left.\log \frac{1}{2}-\log \frac{t-2}{t-1}\right]+\left[\frac{1}{2}-\frac{1}{t-1}\right]\right\}}_{V_{1}}\right. \\
& -\frac{1}{2^{2}} \underbrace{\left[V_{1}+\frac{1}{2}\left(\frac{1}{2^{2}}-\frac{1}{(t-1)^{2}}\right)\right]}_{V_{2}} \\
& \left.+\cdots+\frac{(-1)^{p+1}}{(p+1)^{2}}\left[V_{p}+\frac{1}{p+1}\left(\frac{1}{2^{p+1}}-\frac{1}{(t-1)^{p+1}}\right)\right]+\cdots\right\}, 3 \leqq t \leqq 4 .
\end{aligned}
$$


By means of Newton's method, the explicit expression permits easy calculation of the roots $t_{k}$

$$
v\left(t_{k}\right)=\frac{1}{k}, \quad k=4,5, \cdots, 203 .
$$

For example, the roots

$$
t_{4}=2.1245966, \quad t_{5}=2.2571089
$$

are used by Davenport and Erdös [15].

Centre de Physique Atomique

Université Paul Sabatier

Toulouse, France

1. N. G. DE BRUIJN,"On the number of positive integers $\leqq x$ and free of prime factors $>y$," Nederl. Akad. Wetensch. Proc. Ser. A, v. $54=$ Indag. Math., v. 13, 1951, pp. 50-60. MR 13, 724.

2. N. G. DE BRUIJN, "The asymptotic behaviour of a function occurring in the theory of primes," J. Indian Math. Soc., v. 15, 1951, pp. 25-32. MR 13, 326.

3. K. K. NorTon, "Numbers with small prime factors and least $K$ th power non residue," Mem. Amer. Math. Soc., No. 106, 1971.

4. K. Dickman, "On the frequency of numbers containing prime factors of a certain relative magnitude," Ark. Mat. Astr. Fys., v. 22, A, 1930, pp. 1-14.

5. R. BELlMAN \& B. KotKIN, "On the numerical solution of a differential-difference equation arising in analytic number theory," Math. Comp., v. 16, 1962, pp. 473-475. MR 26 \#5756.

6. J. VAN DE LUNE \& E. WATTEL, "On the numerical solution of a differential-difference equation arising in analytic number theory," Math. Comp., v. 23, 1969, pp. 417-421. MR 40 \#1050.

7. M. P. van Ouwerkerk-DiJKers \& J. Nuis, "On the asymptotic behaviour of the solution of a differential-difference equation arising in number theory," Math. Centrum Amsterdam Afd. Toegepaste Wisk. Rep. TN, v. 50, 1968, 9 pp. MR 41 \#605.

8. L. I. PÁL \& G. NÉMETH, "A statistical theory of lattice damage in solids irradiated by high-energy particles," Nuovo Cimento (10), v. 12, 195.9, pp. 293-309. MR 21 \#7630. 21 \#4264.

9. L. LEWIN, Dilogarithms and Associated Functions, MacDonald, LONDON, 1958. MR

10. J. H. Ahlberg, E. N. Nilson \& J. L. Walsh, The Theory of Splines and Their Applications, Academic Press, New York, 1967. MR 39 \#684.

11. Y. L. LUKE, The Special Functions and Their Approximations. Vol. 2, Math. in Sci. and Engineering, vol. 53, Academic Press, New York, 1969. MR 40 \#2909.

12. E. W. NG, C. J. Devine \& R. F. TOOPER, "Chebyshev polynomial expansion of BoseEinstein functions of orders 1 to 10," Math. Comp., v. 23, 1969, pp. 639-643. MR 40 \#1002a. 13. K. S. KölbIG, "Algorithm 327: Dilogarithm," Comm. Assoc. Comput. Mach., v. 11, 1968 , pp. $270-271$.

14. V. Boffi \& R. Scozzafava, "Sull' equazione funzionale lineare $f^{\prime}(x)=-A(x) f(x-1)$," Rend. Mat. e Appl. (5), v. 25, 1966, pp. 402-410. MR 36 \#1786.

15. H. DAVENPORT \& P. ERDös, "The distribution of quadratic and higher residues," Publ. Math. Debrecen, v. 2, 1951-52, pp. 252-265. 